\title{
Review
}

\section{Cell fate and cell differentiation status in the Arabidopsis root}

\author{
Claudia van den Berg, Peter Weisbeek, Ben Scheres \\ Department of Molecular Cell Biology, Utrecht University, Padualaan 8, NL-3584 CH Utrecht, The Netherlands \\ Received: 5 November 1997 / Accepted: 14 January 1998
}

\begin{abstract}
Post-embryonic development in plants is mainly achieved by its meristems. Within the Arabidopsis root meristem, both the fate and origin of its cells can be predicted with high accuracy. Mutants defective in the determination of root cell fates show that the corresponding genes are first required during embryogenesis. The sub-specification of cell fates, such as during epidermal root hair formation, involves transcription factors and phytohormones. In the Arabidopsis root, initial cell fate specification events must be followed by position-dependent reinforcement of cellular identity. A major question remains as to whether the signals that are involved in initiating the cellular pattern are the same or different from the signals used to reinforce it. The integrity of the root meristem is kept by balancing cell proliferation and cell differentiation, and differentiationinhibiting signals originating from the quiescent centre are involved.
\end{abstract}

Key words: Arabidopsis - Cell differentiation - Cell fate - Root meristem

\section{Introduction}

During embryogenesis of higher organisms, cell types are specified in a specific arrangement during the process of pattern formation. In plants, patterning is not limited to embryogenesis but continues during the adult life cycle as a result of the activity of meristems. Two meristems form during embryogenesis at opposite sides of the apical-basal axis: the shoot apical meristem and the root meristem. In the higher-plant embryo, particular organs are positioned between the meristems: the cotyledons, the hypocotyl and the embryonic root.

Correspondence to: B. Scheres; E-mail: b.scheres@bio.nu.nl; Fax: 31(30)2513655
Perpendicular to the apical-basal axis, different cell types are laid down in concentric layers to form the radial pattern.

Upon germination, new cells and organs arise from the meristems to elaborate the pre-existing cellular pattern of the embryo. The continuation of an embryonic pre-pattern by the meristems is more clearly seen in the root than in the shoot meristem, because the root meristem lacks the complexity of lateral appendage formation.

Here we discuss two issues in plant developmental biology that are currently under intense investigation. We take examples from Arabidopsis root development as this root has proven to be an elegant model system in which to study plant developmental processes (reviewed by Scheres and Wolkenfelt 1997).

The first issue is the mechanisms by which plant cells can acquire and maintain distinct identities. Due to the uncomplicated cellular organization of the Arabidopsis root, in which the developmental history of every cell is known, cell fate specification can be studied at a single cell level (see Figs. 1, 2). Mutants defective in setting up the pattern of cell types in the root are being isolated and their characterization may shed a light on the processes of cell fate specification in plants. Furthermore, in-vivo manipulations involving laser ablations and microinjections, are beginning to provide clues as to mechanisms involved in cell fate specification.

A second issue of current interest is that, within the meristems, cell proliferation occurs in a co-ordinated balance with cell differentiation to maintain a reservoir of undifferentiated cells. Until recently, not much was known about the control of the equilibrium of cell differentiation versus cell proliferation, but recent data from analysis of both root and shoot meristems, provide new insights into this process. In this review, we will highlight data that illustrate new insights into control of cell fate and cell differentiation. Reviews with additional perspectives on root pattern formation and morphogenesis have been recently published elsewhere (Dolan 1996; Malamy and Benfey 1997a; Schiefelbein et al. 1997). 


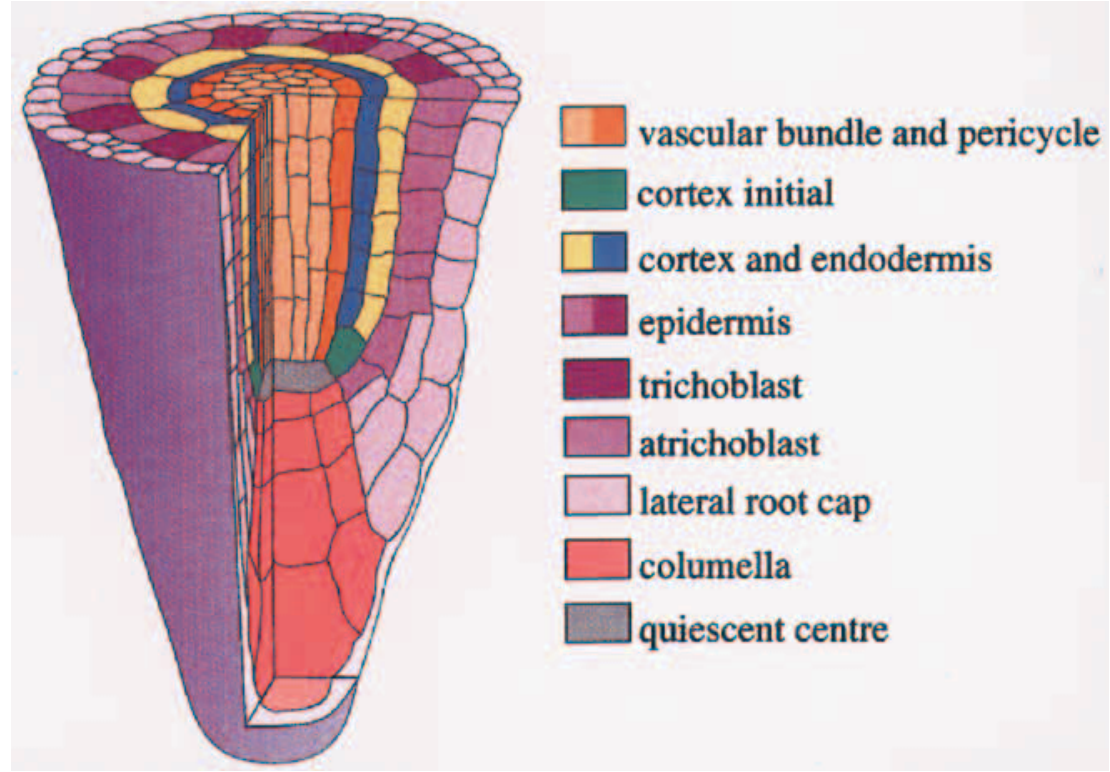

Fig. 1. Schematic representation of the Arabidopsis root

\section{Cellular organization of the Arabidopsis root}

The Arabidopsis root has an uncomplicated cellular organization (Fig. 1; see also Dolan et al. 1993). Within each tissue layer is a low, almost constant, number of cells as viewed in a transverse section. A small vascular bundle, comprising two phloem and two xylem poles in a diarch arrangement, is surrounded by single layers of pericycle, endodermis, cortical parenchyma (cortex hereafter) and epidermis. At the basal end of the root, lateral root cap layers envelop the epidermis. In longitudinal view, the lateral root cap forms continuous files with the columella root cap (Figs. 1, 2c). Files of the different cell types are extended in the meristem by the activity of mitotically active initial cells and their daughters (Fig. 2d). The initials are located basally in the root and are considered to be stem-cell-like cells. They generate new initials which remain in a less differentiated state, and daughter cells which further

Fig. 2a-d. Fate map of the Arabidopsis root. a Globular stage embryo (arrow: first division of the zygote that has generated an apical and a basal cell. b Heart-stage embryo; all cells of the incipient root meristem are present, $\mathbf{c}$ Seedling root, $\mathbf{d}$ Blow-up of central region in root meristem shown in c. Initial cells for all the different cell types surround the quiescent centre. cort, cortex; epid, epidermis; lrc, lateral root cap, col, columella differentiate. Separate sets of initials are present to generate the vascular bundle cells, the pericycle cells and the columella cells. The epidermal initials generate both the epidermis and the lateral root cap, and the cortical initials form the cortex and the endodermis. All initial cells contact the four mitotically inactive cells of the quiescent centre (Fig. 2d). The orientation of cell divisions in the Arabidopsis root meristem is highly invariant and this maintains the overall organization of the root. However, in roots older than a week, differences in cell division patterns cause variation in the cellular organization of the root (Baum and Rost 1996; Rost et al. 1996). First, periclinal divisions in the cortical initials result in the formation of separate initials for cortex and endodermis. Second, the quiescent centre can be activated to divide to form extra cortical cells.

Daughter cells of the initials are gradually displaced from the meristem and they differentiate into specific cell types during that process. For example, in epidermal cells, differentiation leads to the formation of a circumferential pattern consisting of root-hair-bearing and non-hair-bearing cells (Fig. 1; recently reviewed by Dolan 1996, Schiefelbein et al. 1997). However, during the course of cell differentiation, pericycle cells can be reset to initiate new organ formation. These cells re-enter mitosis, resulting in the formation of lateral roots with a similar pattern as the primary root (Dolan et al. 1993).

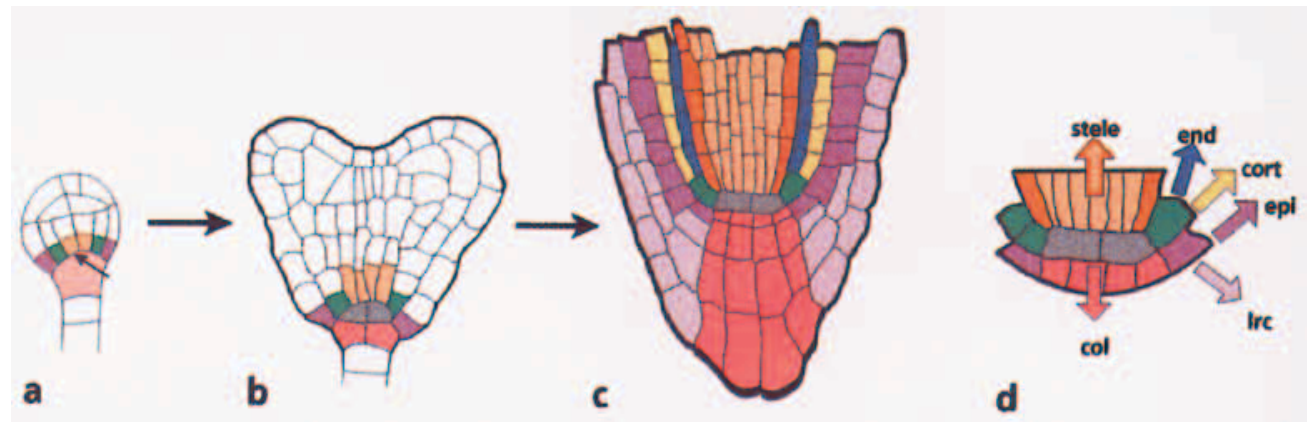



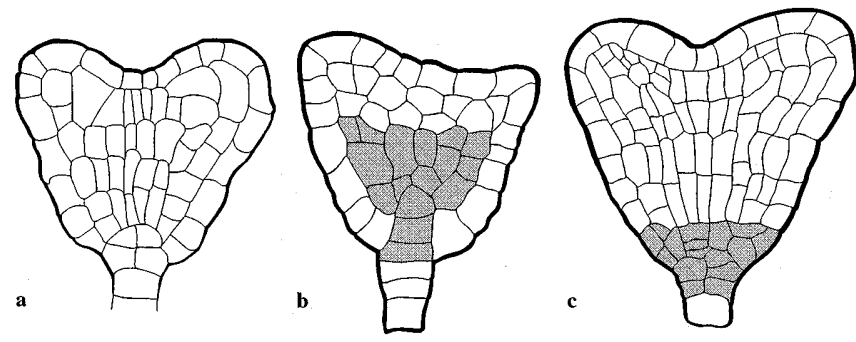

Fig. 3a-d. Schematic representations of heart-stage embryos. a Wild type; b monopteros, a central pile of cells runs in continuity with the suspensor; c hobbit, divisions in the hypophyseal cell derivatives and root meristematic initials are defective; $\mathbf{d}$ scarecrow/shortroot, in both mutants no periclinal divisions in the ground tissue occur. (b redrawn from Przemeck et al. 1996; $\mathbf{c}$ redrawn from Willemsen et al. 1998; a, b, d: early heart stages; c, late heart stage). The affected regions in each mutant are shaded. These do not necessarily represent gene expression domains

In conclusion, the Arabidopsis root has a rather uncomplicated cellular organization, and initial cells and their daughters perpetuate the existing pattern by ordered cell divisions and subsequent differentiation of daughters.

\section{Setting up the pattern}

The cellular organization of the root is established during embryogenesis. In Arabidopsis, the first division of the zygote generates an apical and a basal cell (Fig. 2a, arrow). The root is formed from derivatives of both these cells. The ontogeny of the Arabidopsis root could be described at the cellular level through the analysis of the progeny of genetically marked embryonic cells (Scheres et al. 1994).

The apical-basal pattern within the root results from the ordered development of both apical and basal cell derivatives. The upper derivative of the basal cell, the hypophysis, generates the quiescent centre and the columella root cap initials (Fig. 2b). Apical-cell derivatives make the remainder of the root and root meristem. The cells contacting the hypophysis generate the initials of the root meristem during the heart stage of embryogenesis. The most apical part of the root that connects to the hypocotyl, the collet or embryonic root, is produced by more-apical cells in the embryo.

The radial organization of the root is generated within a group of apical-cell-derived cells at the globular stage of embryogenesis (Fig. 2a). At that stage, cells for the protoderm, ground tissue and procambium (later forming epidermis, cortex/endodermis and vascular bundle, respectively) become separated (Fig. 2b). The mitotic activity of the root meristem initials extends these separate tissue layers basally, from late heart stage onwards.

Hence, an invariant group of embryonic cells participates in the formation of the apical-basal and radial pattern in the root. The separation of different root cell fates occurs during early embryogenesis. It is noteworthy that the separation of the root and hypocotyl occurs

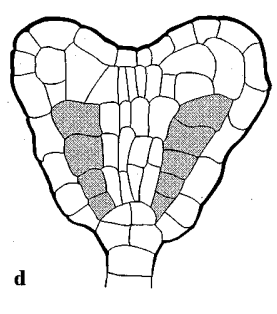

much later during embryonic development and displays some variability: cells around the border can be incorporated either in the root or in the hypocotyl. Their fate is thus dependent on late positional cues.

Several mutant screens have been set up to identify genes involved in determination of cell fates of apicalbasal, radial and circumferential patterning elements of the root. Mutants were isolated on basis of their seedling phenotype (Schiefelbein and Somerville 1990; Benfey et al. 1993; Berleth and Jürgens 1993; Galway et al. 1994; Scheres et al. 1995; Schneider et al. 1997; Willemsen et al. 1998). Below, we describe those that have been characterized in some detail.

Apical-basal pattern formation. A number of mutants have been isolated which lack pattern elements in the apical-basal axis of the embryo (Mayer et al. 1991; Scheres et al. 1996). Mutations in the MONOPTEROS $(M P)$ gene lead to seedlings lacking both root and hypocotyl (Berleth and Jürgens 1993). The defect in $m p$ mutants is evident as early as the octant stage of embryogenesis. At the triangular stage, cells of both the lower tier and the hypophysis divide aberrantly. The inner cells of the lower tier, which make the vascular cells of root and hypocotyl, fail to produce elongated cell files. Furthermore, a pile of cells in the centre forms by horizontal divisions, running in continuity with the suspensor (Fig. 3b).

The capacity of $m p$ mutants to make adventitious roots was used to study the post-embryonic requirements for the $M P$ gene product. The $m p$ plants can make largely normal apical structures. All organs, however, display defective vascular strands that are disrupted (Przemeck et al. 1996). Furthermore, auxin transport capacity in inflorescence axes is impaired. These observations led us to the conclusion that MP, rather than specifying apical-basal pattern elements, promotes cell axialization and cell file formation which is important for both embryonic axis formation and vascular system development.

Cell fate determination along the apical-basal axis also involves the $H B T$ gene. The hbt mutants have cotyledons, a hypocotyl and a small embryonic root. However, no specification of the meristematic root occurs (Willemsen et al. 1998). Lateral and adventitious roots also form abnormally in hbt. The mutational defect first becomes apparent around the quadrant stage of embryogenesis. From that stage onwards, atypical divisions occur in the hypophyseal progenitor cell (Fig. 3c). This defect correlates precisely with the absence of an anatomically recognizable quiescent centre 
and the lack of columella root cap cell specification in $h b t$ seedlings. After the heart stage of embryogenesis, the divisions of the cells occupying the position of the initial cells in the wild type are strongly reduced or completely absent in hbt mutants (Fig. 3c). Furthermore, in the region where normally the lateral root cap is formed, divisions are defective. These defects lead to seedlings without a functional root meristem. The $H B T$ gene could thus be involved in specifying the hypophyseal cell derivatives, the root meristematic initials and the lateral root cap. Alternatively, $H B T$ could be involved primarily in specifying the hypophyseal cell. Subsequently, $H B T$-expressing cells could signal to the directly contacting apical cells to initiate meristematic mitotic initial activity and lateral root cap specification. In the first case, $H B T$ would be functional in the complete root meristem. In the latter, $H B T$ would be functional in the hypophyseal cell derivatives only. The $h b t$ seedlings are also impaired in cell expansion and epidermal cell development in regions not affected in the embryo, suggesting additional post-embryonic requirements for the HBT gene.

Radial pattern formation. Several mutants have been described lacking radial pattern elements in the root (Benfey et al. 1993; Scheres et al. 1995). Both scarecrow (scr) and shortroot (shr) lack a layer of the ground meristem (Fig. 3d). The shr mutants lack an endodermis whereas the remaining ground-tissue layer in scr mutants expresses both cortical and endodermal markers (Scheres et al. 1995; Di Laurenzio et al. 1996). Both mutant phenotypes are first manifested at the heart stage of embryogenesis where the periclinal division that doubles the ground meristem does not occur in the root or in the hypocotyl (Fig. 3d). To determine whether the shr and scr mutants are primarily disturbed in cell division in the ground tissue, or primarily in fate specifications of the ground tissue, double mutants with fass were made (Scheres et al. 1995). The fass mutants display additional cell divisions in all cell layers (Torres-Ruiz and Jürgens 1994). Double mutants of $s h r$ and fass have more ground-tissue layers. However, an endodermis-specific cell layer could not be restored. Considering these observations, it was concluded that SHR primarily specifies endodermal cell fate. In contrast, scr and fass double mutants have a single endodermal layer surrounded by multiple layers lacking endodermal attributes. Apparently, SCR is involved in controlling the periclinal division in the ground meristem that leads to separate cortical and endodermal cell layers. Recently, the $S C R$ gene has been cloned (Di Laurenzio et al. 1996). It encodes a novel putative transcription factor, and it is not known how the gene product controls cell division. It is likely that other, SCR-dependent, genes are involved in the process that ultimately leads to the periclinal division in the ground meristem. In-situ hybridization and an enhancer-trap insertion upstream of the $S C R$ gene reveal that $S C R$ is expressed in the cortical initial and in all endodermal cells. This contrasts with the assumption that SCR regulates the asymmetric division in the cortical initial only, to separate cortex and endodermis.
More work is needed to determine the functional relevance of the endodermal expression of $S C R$. It is possible that $S C R$ is required both for the division in the cortical initials to separate cortex and endodermis, and for endodermal fate specification or maintenance.

A mutation in the WOODEN LEG (WOL) gene results in seedlings with fewer vascular cells in root and hypocotyl, without phloem specification (Scheres et al. 1995). Enhancing the amount of cells (in a fass background) restores phloem formation. This indicates that the wol mutant primarily interferes with cell division, with a lack of specific cell fates as a secondary consequence, rather than being involved primarily in cell fate specification. The amount of cells available seems to be critical at the period during which cell types are specified. In wol mutants, the formation of xylem cells may use up all the available cells and thus no phloem forms. This 'first-come-first-served' mechanism was also suggested for the action of WUSCHEL in floral meristems (Laux et al. 1996). The WUSCHEL gene is required for the identity of the central zone in shoot and floral meristems. In wuschel mutant flowers, fewer than the normal number of cells are present, and third- and fourth-whorl organs are not formed.

Taken together, mutant screens based solely on seedling phenotypes have revealed a limited number of mutants affecting the radial organization in the root, and these confirm the notion that the cellular pattern of the root is set up in the embryo. The screens, however, are based on the overall appearance of the root (e.g. root length). Some mutants defective in the radial organization of the root may be very subtle, and have no effects on overall root growth. Furthermore, these screens fail to detect a conceivable class of embryo-lethal pattern mutants. Additional screens using $\beta$-glucuronidase or Green Fluorescent Protein coupled to tissue specific promoters, to mark the presence of cell types and hence enable genetic screens for ectopic presence or loss of these cell types, might unravel more players in the process of radical pattern formation in the future.

Circumferential pattern formation. Once outside the meristem, cells further elongate and differentiate. Epidermal cells have the ability to form root hairs. In the Arabidopsis seedling root, files of hair-bearing (trichoblast) cells alternate with files of hairless (atrichoblast) cells. The trichoblast cells are located over the cell wall clefts between two underlying cortical cells whereas nonhair cells only contact a single cortical cell (Dolan et al. 1994). It is thought that the occurrence of radial walls in underlying cortical cells determines, at least in part, epidermal cell fate (see below). The difference between trichoblast cells and atrichoblast cells is already visible prior to the outgrowth of the hair. Trichoblasts are more elongated, have a more densely stained cytoplasm and have a delayed vacuolation (Galway et al. 1994).

Mutant analyses have identified genes involved in root hair formation (Table 1). The mutants transparent testa glabra (ttg), glabra2 (gl2), ectopic root hairl (erhl), ectopic root hair2 (erh2) and ectopic root hair3 (erh3) have root hairs on almost all epidermal cells (Galway 
et al. 1994; Masucci et al. 1996; Schneider et al. 1997). In ttg, erhl and erh3 mutants, all morphological differences between atrichoblast and trichoblast cells are lost, whereas in $g l 2$ and erh2 mutants some morphological differences between these two cell types still exist. GL2 encodes a putative homeodomain-containing transcription factor and is expressed in all atrichoblasts, except in the epidermal initials and their first and second daugh- ters (Rerie et al. 1994; Masucci et al. 1996). In $t$ tg mutants, GL2 expression is strongly reduced, suggesting that in wild-type $T T G$ is a positive regulator of GL2 (Di Christina et al. 1996).

The caprice (cpc) mutants have fewer root hairs than the wild type. Recently, the $C P C$ gene was cloned (Wada et al. 1997). It encodes a protein with a Myb-like DNAbinding domain. Double mutant combinations with GL2

Table 1. Mutants defective in cell fate specification in Arabidopsis roots discussed in this review. WT, wild type

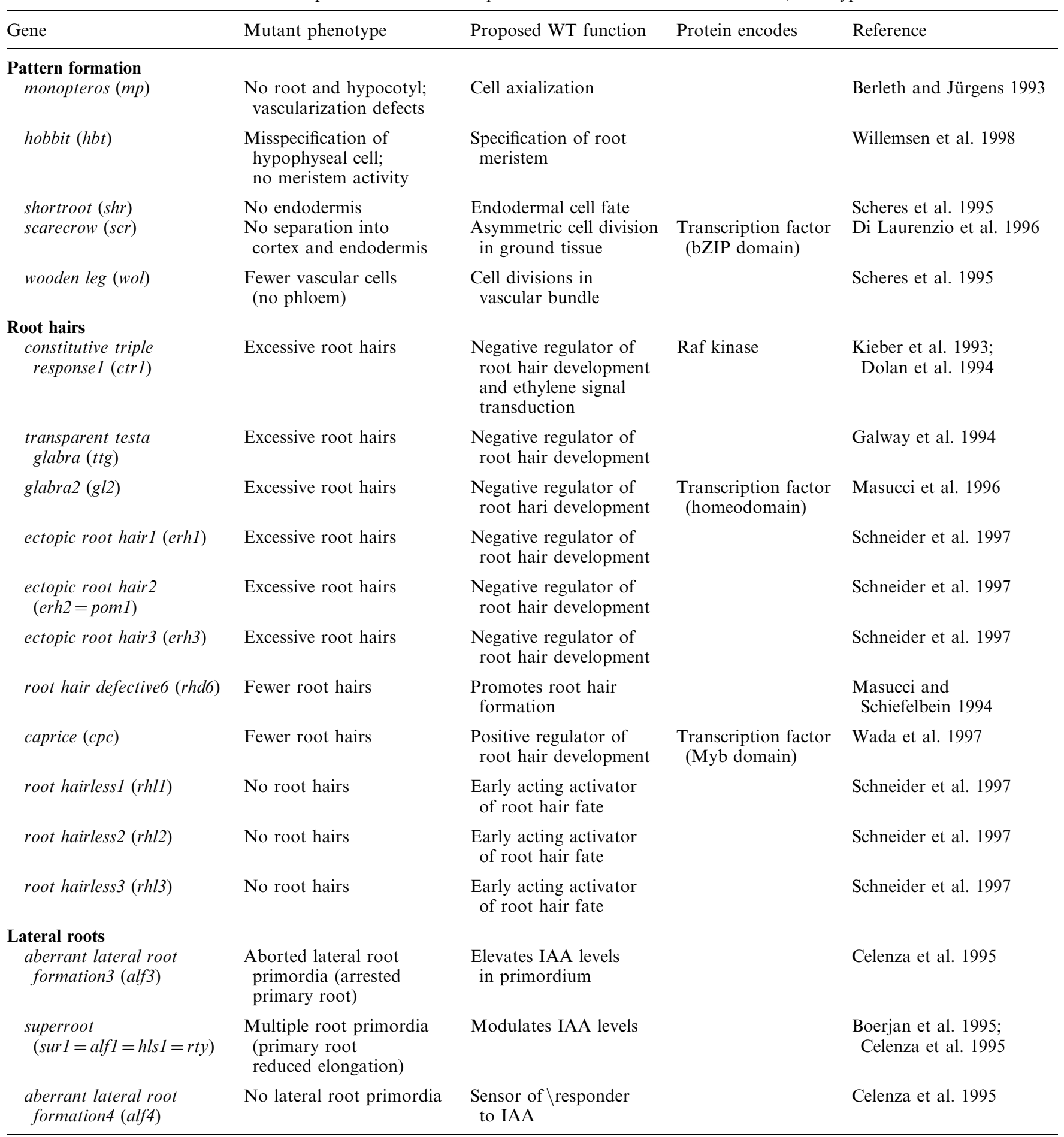


suggest that $C P C$ may inhibit GL2. As the expression pattern of $C P C$ was not determined, it is unknown in which epidermal cells $C P C$ is active and thus whether it is possible that CPC can directly inhibit GL2.

Ethylene plays a role in root epidermal fate and promotes the formation of root hairs (Tanimoto et al. 1995; Masucci and Schiefelbein 1996). Germination of plants in the presence of 1-amino-1-cyclopropane carboxylate (ACC), an ethylene precursor, results in ectopic hair formation. Addition of 2-aminoethoxyvinyl glycine (AVG), an inhibitor of ACC formation, leads to a decrease in root hairs.

A number of mutations in the ethylene response pathway have been implicated in epidermal cell patterning. One of these genes, CONSTITUTIVE TRIPLE RESPONSEl (CTRl) encodes a RAF-like kinase and is proposed to negatively regulate ethylene signalling (Kieber et al. 1993). The ctrl mutants form root hairs on epidermal cells that are normally hairless (Dolan et al. 1994). Furthermore, ROOT HAIR DEFECTIVE ( $R H$ D6) mutations cause a strong reduction in root hair formation and the mutant phenotype can be suppressed by ACC (Masucci and Schiefelbein 1994). The root hairless1 (rhll), root hairless2 (rhl2) and root hairless3 (rhl3) mutants lack root hairs. Double mutant analysis and ethylene addition experiments indicate that the RHL genes act before $C T R 1$, the $E R H$ genes, ethylene and probably before $T T G$ and GL2 (Schneider et al. 1997). Together with the loss of morphological differences between trichoblasts and atrichoblasts in the mutants, these data suggest that the $R H L$ genes play the earliest role in epidermal cell fate specification analyzed so far. The rhl mutants also have defects in shoot development which suggest that the corresponding gene products might have more than one role in plant development.

It was shown that the expression of GL2 is not altered by the presence of ethylene or in a rhd6 or ctr 1 mutant background (Masucci and Schiefelbein 1996). Double mutant analysis indicated that RHD6 is negatively regulated by, or acts separately from, TTG and GL2. These data may imply that TTG and GL2 act upstream of ethylene by negatively regulating the ethylene signalling pathway. It was proposed that the position-dependent pattern of epidermal cell types is controlled by TTG and GL2, which results in repression of the ethylene signal transduction pathway (Masucci and Schiefelbein 1996). In addition, epidermal cells may receive different amounts of ethylene, resulting in a differential activation of the ethylene signalling cascade (Dolan et al. 1994).

In conclusion, mutant analyses begin to shed some light on the processes of fate determination in the root, most notably in the case of epidermal sub-specification. However, these analyses do not reveal at this moment how the meristematic pattern is perpetuated and we are still far away from understanding cell specification at the molecular level.

\section{Formation of lateral roots}

How are distinct identities of cells acquired and elaborated, in non-embryogenic roots? Does pattern formation require the same signals in both primary and lateral roots? Lateral roots are formed after embryogenesis and their anatomy is highly similar to that of primary roots. Lateral roots arise from pericycle cells that re-enter mitosis. The addition of exogenous auxin dramatically increases the number of lateral roots (Celenza et al. 1995 and refs. therein). The superroot (syn, aberrant lateral root formation1 syn hookless3) mutants, which have an increased indole acetic acid (IAA) level, display a similar phenotype (Boerjan et al. 1995; Celenza et al. 1995). Moreover, auxin-resistant mutants have a reduced number of lateral roots (Hobbie and Estelle 1995; Timpte et al. 1995). Other mutants defective in lateral root formation seem to be insensitive to application of IAA. In aberrant lateral root formation4 (alf4) mutants, lateral root formation is not stimulated by the addition of IAA or the introduction of mutants with elevated IAA levels in an alf4 mutant background. Although it clearly serves an important role, the mechanism by which auxin induces lateral root formation is unknown. The alf4 mutants fail to initiate lateral roots but the primary root develops normally (Celenza et al. 1995). On the other hand, $m p$ mutants make normal secondary roots, indicating that MP is strictly required for root initiation in the embryo only (Berleth and Jürgens 1993).

Upon initiation, however, mutant analyses and the expression patterns of tissue specific markers suggest that similar mechanisms of pattern formation are used to further set up and elaborate the pattern in roots derived from different origins (Willemsen et al. 1998; Scheres et al. 1995; Malamy and Benfey 1997b). Mutations in $H B T$, $S H R, S C R$ and $W O L$ display the same defects in both primary and lateral roots (Willemsen et al. 1998; Scheres et al. 1995). In conclusion, it is likely that lateral root initiation is regulated differently from primary root initiation. However, once initiated, pattern formation appears to be regulated by a similar set of genes.

Lateral root primordia can, from early stages onwards, be excised and grown further (Laskowski et al. 1995). Furthermore, marker genes that are expressed early in lateral root formation appear to have comparable expression patterns in the embryo (compare Di Laurenzio et al. 1996 with Malamy and Benfey 1997b). Mutant screens based on altered expression patterns of marker genes in lateral roots are less laborious than screening for altered or missing expression patterns in embryos. Studying lateral roots may thus be a promising approach to enhance our knowledge on cell fate specification during formation of the less accessible primary root.

\section{Position determines cell fate in the root meristem}

Because the root forms no lateral extensions within the meristem, one can clearly observe that once the 
meristematic organization is established, the resulting pattern is continuously perpetuated. New cells are added to the root by the activity of initials and their daughters. How is the cellular pattern perpetuated? How do cells within the meristem know when and how to divide and into which cell type they should differentiate? The rigid clonal relationship between meristematic cells and their ancestors in the Arabidopsis root can be taken to indicate that cell fate determinants are distributed early, with no or only limited flexibility for switching fate at later stages. However, surgical experiments in various meristems showed that meristematic cells can be very flexible (reviewed by Steeves and Sussex 1989). Accordingly, laser ablations in the Arabidopsis root meristem showed that cells change fate upon changing position (van den Berg et al. 1995). Ablation of cells results in their shrinking and the available space becomes occupied by neighbouring cells. For instance, ablation of a cortical initial leads to the invasion of pericycle cells. These pericycle cells start producing cortex and endodermis in accordance with their new position. Isolation of a cortical initial from its daughters perturbs its specific differentiation, indicating that positional cues originate from older cells of their own cell type (van den Berg et al. 1995). Cells thus differentiate similarly to their more apical daughters. The molecular nature of these positional cues is thus far unknown and it is not clear how they are distributed.

Recently, the mapping of plasmodesmata and dyeloading experiments have indicated that plasmodesmata are preferentially located within files of the same cell type and not between different cell types (Oparka et al. 1994; T. Zhu, W. Lucas, and T.L. Rost, UCA Davis, USA. personal communications). It is thus well conceivable that positional signals are targeted via plasmodesmata. Support for this suggestion comes from fluorescent dye injections in the epidermis. The dye can move to other epidermal cells but not to cells of other cell types (Duckett et al. 1994). Additional evidence for the importance of plasmodesmatal transport comes from studies on fate determination in the shoot meristem. KNOTTED-1 mRNA is localized in all cells of the shoot meristem except the outermost layer (L1 layer; Jackson et al. 1994). The KNOTTED-1 protein, however, is present in all cells including the L1 layer. Injection experiments with fluorescently labelled KNOTTED-1 protein and its mRNA showed that KNOTTED-1 can traffic between cells and that it facilitates the movement of KNOTTED-1 mRNA to other cells (Lucas et al. 1995). Ablation experiments disturb plasmodesmatal connections, but the possibility of rapid re-formation of plasmodesmata in new neighbours does not preclude a role for plasmodesmatal transport in respecification events upon laser ablation.

Alternatively, cell fate could be determined by the selective depositing of signalling molecules in the cell wall. Laser ablations in the brown alga Fucus have shown that cell wall components can be instructive in cell fate decisions (Berger et al. 1994). The laser ablation experiments in the Arabidopsis root do not provide information about whether cell walls are instrumental in fate determination, since short-lived wall molecules may not persist in walls surrounding corpses of ablated cells. The identification and analysis of molecular components involved in signalling are required to determine whether positional cues guiding root cell fate are targeted via plasmodesmata or via the cell wall.

The laser ablation experiments show that the root meristem acts to copy the existing embryonic pattern, which can compensate for variations in meristematic cell division patterns. It is possible that the same signals that are active in embryogenic patterning, also function postembryonically as continuous positional cues. Alternatively, different signals can be used in setting up and in perpetuating the cellular pattern.

\section{The regulation of cell proliferation and differentiation}

A proper balance between cell proliferation and cell differentiation in meristems is necessary to guarantee continuous development. If signals guiding cell fate resulted in progression of cell differentiation in the initial cells, a determined meristem would be the result. What keeps initial cells from differentiating? It is noteworthy that initials can not be considered as undifferentiated cells. Certain cell-type-specific promoter/enhancer trap constructs show expression in a complete tissue, including its initials (e.g. van den Berg et al. 1995). Therefore, the progression of differentiation should be regulated and not the maintenance of a group of completely undifferentiated cells.

Ablation of a quiescent-centre cell results in the progression of differentiation of contacting columella initials. These initials now express a marker that is normally only expressed in more-mature columella cells (van den Berg et al. 1997). In contacting cortical initials,

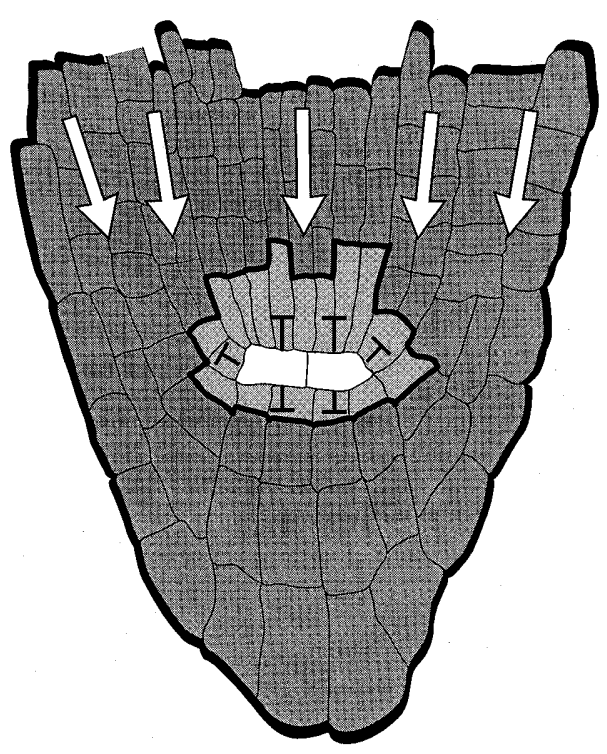

Fig. 4. A simple model representing two different regulatory signals within the root meristem. The quiescent centre inhibits differentiation of surrounding initials, whereas positional cues direct differentiation into different cell types 
progression of differentiation was also observed after quiescent-centre ablation, resulting in the division of cortical initials in a 'daughter-specific' manner. Thus, the quiescent centre appears to inhibit the progression of differentiation of surrounding cells in the root (Fig. 4). Inhibition of differentiation only occurs in cells that contact the quiescent centre, which reveals that shortrange or contact-dependent signals are involved in this process. It is noteworthy that all the initial cells contact the quiescent centre. In conclusion, the quiescent centre plays an important role in controlling the balance between cell proliferation and cell differentiation, although additional controls may be involved.

In the shoot meristem, mutants have been characterized that are disturbed in cell proliferation and/or differentiation. Mutations in the CLAVATAI and CLA$V A T A 3$ genes alter the balance of cell proliferation versus cell differentiation (Clark et al. 1993, 1995). The clavata mutants have enlarged shoot and floral meristems caused by excessive accumulation of undifferentiated cells. The gene CLAVATAl encodes a putative receptor kinase and is expressed in the central undifferentiated cells and in a portion of cells contributing to organ formation (Clark et al. 1997). Studies on the clavata mutants have not yet revealed whether CLAVATA inhibits cell division of stem cells or whether it promotes incorporation of differentiated cells into organs.

Root meristems also contain a central zone of less differentiated cells. It will be interesting to find out whether $C L A V A T A$-like genes play a role in the control of cell proliferation and cell differentiation in a similar manner in the root as in the shoot meristem.

\section{Concluding remarks}

Initiation of the cellular pattern during embryogenesis and perpetuation of this pattern during seedling development, and regulation of the balance between cell proliferation and cell differentiation are two important aspects of root development. Regarding the first aspect, several mutants have been identified with specific defects in the root cellular pattern. It turns out that the corresponding genes are first required during the early stages of embryo development, and they are required throughout the embryonic axis. Circumferential patterning seems to involve pre-patterned transcription factors (such as GL2), and phytohormones (most notably ethylene), which may be differentially allocated to or received by epidermal cells. Comparisons of mutant phenotypes and marker-gene expression patterns indicate that primary root formation probably involves the same mechanisms as secondary root formation. To construct a better genetic framework for cell specification in the Arabidopsis root, novel mutant screening strategies will be required. The use of markers for specific cell types in primary or lateral roots will be useful in these screens to identify novel genes involved in pattern formation.

Laser ablation experiments indicate that positional information continuously acts to reinforce the specifica- tion of newly added cells in the root meristem. Mutant analysis has not yet provided a genetic entrance in the nature of these positional signals. Future challenges will be to resolve both the nature of the positional cues and the mechanisms to receive these cues.

In the root meristem, signals derived from the quiescent centre keep the surrounding initial cells in a less differentiated state. It will be interesting to determine whether there is an interplay between quiescent-centrederived signals inhibiting differentiation, and positional signals promoting cell-type-specific differentiation.

Molecular genetic and surgical approaches will ultimately lead to a more complete picture of how the Arabidopsis root develops. Although it is likely that major developmental mechanisms are conserved, it will be both important and interesting to find out whether the many different types of root apices described in angiosperms (Barlow 1976; Feldman 1984; Abeysekera and McCully 1993; Clowes 1994) utilize similar mechanisms for pattern formation and meristem maintenance.

We thank Frouke Kuyer for excellent art work. C.v.d.B. was sponsored by a grant from the Dutch Organisation of Life Sciences (SLW).

\section{References}

Abeysekera RM, McCully ME (1993) The epidermal surface of the maize root tip. New Phytol 125: 413-429

Barlow PW (1976) Towards an understanding of the behaviour of root meristems. J Theor Biol 57: 433-451

Baum SF, Rost TL (1996) Root apical organization in Arabidopsis thaliana 1. Root cap and protoderm. Protoplasma 192: 178-188

Benfey PN, Linstead PJ, Roberts K, Schiefelbein JW, Hauser M-T, Aeschbacher RA (1993) Root development in Arabidopsis: four mutants with dramatically altered root morphogenesis. Development 119: 57-70

Berger F, Taylor A, Brownlee C (1994) Cell fate determination by the cell wall in early Fucus development. Science 263: 1421-1423

Berleth T, Jürgens G (1993) The role of the monopteros gene in organising the basal body region of the Arabidopsis embryo. Development 118: 575-587

Boerjan W, Cervera M-T, Delarue M, Beeckman T, Dewitte W, Bellini C, Caboche M, van Onckelen H, van Montagu M, Inzé D (1995) superroot, a recessive mutation in Arabidopsis, confers auxin overproduction. Plant Cell 7: 1405-1419

Celenza JLJr, Grisafi PL, Fink GR (1995) A pathway for lateral root formation in Arabidopsis thaliana. Genes Dev 9: 2131-2142

Clark SE, Running MP, Meyerowitz EM (1993) CLAVATA1, a regulator of meristem and flower development in Arabidopsis. Development 119: 397-418

Clark SE, Running MP, Meyerowitz EM (1995) CLAVATA3 is a specific regulator of shoot and floral meristem development affecting the same processes as CLAVATA1. Development 121: 2057-2067

Clark SE, Williams RW, Meyerowitz EM (1997) The CLAVATA1 gene encodes a putative receptor kinase that controls shoot and floral meristem size in Arabidopsis. Cell 89: 575-585

Clowes FAL (1994) Origin of the epidermis in root meristems. New Phytol 127: 335-347

Di Christina M, Sessa G, Dolan L, Linstead P, Baima S, Ruberti I, Morelli G (1996) The Arabidopsis Athb-10 (GLABRA2) is an HD-Zip protein required for regulation of root hair development. Plant J 10: 393-402 
Di Laurenzio L, Wysocka-Diller J, Malamy JE, Pysh L, Helariutta Y, Freshour G, Hahn MG, Feldmann KA, Benfey PN (1996) The SCARECROW gene regulates an asymmetric cell division that is essential for generating the radial organization of the Arabidopsis root. Cell 86: 423-433

Dolan L, Janmaat K, Willemsen V, Linstead P, Poethig S, Roberts K, Scheres B (1993) Cellular organisation of the Arabidopsis thaliana root. Development 119: 71-84

Dolan L, Duckett CM, Grierson C, Linstead P, Schneider K, Lawson E, Dean C, Poethig S, Roberts K (1994) Clonal relationships and cell patterning in the root epidermis of Arabidopsis. Development 120: 2465-2474

Dolan L (1996) Pattern in the root epidermis: an interplay of diffusible signals and cellular geometry. Ann Bot 77: 547-553

Duckett CM, Oparka KJ, Prior DAM, Dolan L, Roberts K (1994) Dye-coupling in the root epidermis of Arabidopsis is progressively reduced during development. Development 120: 32473255

Feldman LJ (1984) Regulation of root development. Annu Rev Plant Physiol 35: 223-242

Galway M, Masucci J, Lloyd A, Walbot V, Davis R, Schiefelbein J (1994) The $T T G$ gene is required to specify epidermal cell fate and cell patterning in the Arabidopsis root. Dev Biol 166: 740-754

Hobbie L, Estelle M (1995) the axr4 auxin-resistant mutants of Arabidopsis thaliana define a gene important for root gravitropism and lateral root initiation. Plant J 7: 211-220

Jackson D, Veit B, Hake S (1994) Expression of maize KNOTTED-1 related homeobox genes in the shoot apical meristem predicts patterns of morphogenesis in the vegetative shoot. Development 120: $405-413$

Kieber JJ, Rothenberg M, Roman G, Feldmann KA, Ecker JR (1993) CTR1, a negative regulator of the ethylene response pathway in Arabidopsis, encodes a member of the Raf family of protein kinases. Cell 72: 427-441

Laskowski MJ, Williams ME, Nusbaum HC, Sussex IM (1995) Formation of lateral root meristems is a two-stage process. Development 121: 3303-3310

Laux T, Mayer KFX, Berger J, Jürgens G (1996) The WUSCHEL gene is required for shoot and floral meristem integrity in Arabidopsis. Development 122: 87-96

Lucas WJ, Bouché-Pillon S, Jackson DP, Nguyen L, Baker L, Ding B, Hake S (1995) Selective trafficking of KNOTTED-1 homeodomain protein and its mRNA through plasmodesmata. Science 270: 1980-1983

Malamy JE, Benfey PN (1997a) Down and out in Arabidopsis: the formation of lateral roots. Trends Plant Sci 2: 390-396

Malamy JE, Benfey PN (1997b) Organization and cell differentiation in lateral roots of Arabidopsis thaliana. Development 124: 33-44

Masucci JD, Schiefelbein JW (1994) The rhd6 mutation in Arabidopsis thaliana alters root-hair initiation through an auxin- and ethylene-associated process. Plant Physiol 106: 1335-1346

Masucci JD, Rerie WG, Foreman DR, Zhang M, Galway ME, Marks DM, Schiefelbein JW (1996) The homeobox gene GLABRA2 is required for position-dependent cell differentiation in the root epidermis of Arabidopsis thaliana. Development 122: $1253-1260$

Masucci JD, Schiefelbein JW (1996) Hormones act downstream of $T T G$ and $G L 2$ to promote root hair outgrowth during epidermis development in the Arabidopsis root. Plant Cell 8: 1505-1517
Mayer U, Torres-Ruiz RA, Berleth T, Miséra S, Jürgens G (1991) Mutations affecting body organization in the Arabidopsis embryo. Nature 353: 402-407

Oparka KJ, Duckett CM, Prior DAM, Fisher DB (1994) Real-time imaging of phloem unloading in the root tip of Arabidopsis. Plant J 6: 759-766

Przemeck GKH, Mattsson J, Hardtke CS, Sung ZR, Berleth T (1996) Studies on the role of the Arabidopsis gene MONOPT$E R O S$ in vascular development and plant cell axialization. Planta 200: 229-237

Rerie WG, Feldmann KA, Marks MD (1994) The GLABRA2 gene encodes a homeodomain protein required for normal trichome development in Arabidopsis. Genes Dev 8: 1388-1399

Rost TL, Baum SF, Nichol S (1996) Root apical organization in Arabidopsis thaliana ecotype 'WS' and a comment on root cap structure. Plant Soil 187: 91-95

Scheres B, Wolkenfelt H, Willemsen V, Terlouw M, Lawson E, Dean C, Weisbeek P (1994) Embryonic origin of the Arabidopsis primary root and root meristem initials. Development 120: 2475-2487

Scheres B, Di Laurenzio L, Willemsen V, Hauser M-T, Janmaat K, Weisbeek P, Benfey PN (1995) Mutations affecting the radial organisation of the Arabidopsis root display specific defects throughout the embryonic axis. Development 121: 53-62

Scheres B, McKhann H, van den Berg C, Willemsen V, Wolkenfelt H, de Vrieze G, Weisbeek P (1996) Experimental and genetic analysis of root development in Arabidopsis thaliana. Plant Soil 187: $97-105$

Scheres B, Wolkenfelt H (1997) The Arabidopsis root as a model to study plant development. Plant Physiol Biochem, in press

Schiefelbein JW, Somerville C (1990) Genetic control of root hair development in Arabidopsis thaliana. Plant Cell 2: 235-243

Schiefelbein JW, Masucci JD, Wang H (1997) Building a root: the control of patterning and morphogenesis during root development. Plant Cell 9: 1089-1098

Schneider K, Wells B, Dolan L, Roberts K (1997) Structural and genetic analysis of epidermal cell differentiation in Arabidopsis primary roots. Development 124: 1789-1798

Steeves TA, Sussex IM (1989) Patterns of plant development. 2nd edn. Cambridge University Press, New York

Tanimoto M, Roberts K, Dolan L (1995) Ethylene is a positive regulator of root hair development in Arabidopsis thaliana. Plant J 8: 943-948

Timpte C, Lincoln C, Pickett FB, Turner J, Estelle M (1995) The $A X R I$ and $A U X 1$ genes of Arabidopsis function in separate auxin-response pathways. Plant J 8: 561-569

Torres-Ruiz RA, Jürgens G (1994) Mutations in the FASS gene uncouple pattern formation and morphogenesis in Arabidopsis development. Development 120: 2967-2978

van den Berg C, Willemsen V, Hage W, Weisbeek P, Scheres B (1995) Cell fate in the Arabidopsis root meristem determined by directional signalling. Nature 378: 62-65

van den Berg C, Willemsen V, Hendriks G, Weisbeek P, Scheres B (1997) Short-range control of cell differentiation in the Arabidopsis root. Nature 390: 287-289

Wada T, Tachibana T, Shimura Y, Okada K (1997) Epidermal cell differentiation in Arabidopsis determined by a Myb-homolog, CPC. Science 277: 1113-1116

Willemsen V, Wolkenfelt H, de Vrieze G, Weisbeek P, Scheres B (1998) The hobbit gene is required for formation of the root meristem in the Arabidopsis embryo. Development, in press 